\title{
Approaching the Square of Oppositions in Terms of the $f$-indexes of Inclusion and Contradiction
}

\author{
Nicolás Madrid and *Manuel Ojeda-Aciego \\ Departamento Matemática Aplicada, Universidad de Málaga \\ \{nicolas.madrid, aciego\}@uma.es
}

\begin{abstract}
We continue analyzing the properties of $f$ inclusion and $f$-contradiction, and provide an alternative interpretation of the square of opposition.
\end{abstract}

Keywords: Fuzzy sets, Opposition square, $f$-Inclusion, $f$-Contradiction

\section{Introduction}

The square of opposition admits interpretations in terms of several logic frameworks, from classical logic (both propositional and first-order) to various modal logic approaches, even a gradual generalization has been introduced.

We focus here on an initial interpretation of the square of opposition in terms of the functional approach to graded inclusion and contradiction, which proposes using certain mappings in the unit interval ( $f$-indexes) and leads to the notions of degree of $f$-inclusion $[8,9]$ and degree of $f$-contradiction [3].

The paper is structured as follows: in Section 2 we recall some basic notions of fuzzy set theory, the notion of $f$-inclusion and the notion of $f$-weak-contradiction. In Section 3 we introduce some properties of the $f$ degrees of inclusion and of contradiction and, then, provide a first approach towards an interpretation of the square of opposition. Finally, in Section 4 we present conclusions and future works.

\section{Preliminary definitions}

Let us recall that a fuzzy set $A$ is defined on a referential universe $\mathscr{U}$ (usually omitted) by means of its membership function $A: \mathscr{U} \rightarrow[0,1]$. The standard operations union and intersection between sets can be extended to operations between fuzzy sets as follows: given two fuzzy sets $A$ and $B$, we define the fuzzy sets $A \cup B$ and $A \cap B$ as $A \cup B(u)=\max \{A(u), B(u)\}$ and $A \cap B(u)=$ $\min \{A(u), B(u)\}$ for all $u \in \mathscr{U}$, respectively. To define the complement of a fuzzy set we need to consider negation operators. Let us recall that a negation operator is a decreasing mapping $n:[0,1] \rightarrow[0,1]$ such that $n(0)=1$ and $n(1)=0$. Given a fixed negation operator, $n$, the complement of a fuzzy set $A$ is defined as $A^{c}(u)=n(A(u))$ for all $u \in \mathscr{U}$. We say that a negation $n$ is involutive if $n(n(x))=x$ for all $x \in[0,1]$. Note that in the case of considering an involutive negation for the definition of the complement, the double complement law holds; i.e., $\left(A^{c}\right)^{c}=A$.

\subsection{Functional degrees of inclusion and contradiction}

The main difference of our approach with respect to other approaches dealing with measures of inclusion between fuzzy sets $[7,11,13,14]$, is that the degrees used to express the measure are no longer real values, but certain mappings from $[0,1]$ to $[0,1]$. These mappings should satisfy the properties of deflation and monotonicity in order to be considered indexes of inclusion.

Definition 1 The set of $f$-degrees of inclusion (denoted by $\Omega$ ) is the set of mappings $f:[0,1] \rightarrow[0,1]$ satisfying the following properties for all $x, y \in[0,1]$ :

- $f(x) \leq x$

- if $x \leq y$ then $f(x) \leq f(y)$

The definition of $f$-inclusion [9] is given as follows.

Definition 2 Let $A$ and $B$ be two fuzzy sets and consider $f \in \Omega$. We say that $A$ is $f$-included in $B$ (denoted by $A \subseteq_{f} B$ ) if and only if the inequality $f(A(u)) \leq B(u)$ holds for all $u \in \mathscr{U}$. 
It is worth remarking that, different mappings in $\Omega$ define different relations of $f$-inclusion that determine stronger or weaker restrictions.

$\Omega$ has a lattice structure with the usual pointwise ordering between mappings. We will use id and $\perp$ to refer to the greatest and lowest mappings in $\Omega$; i.e., $i d(x)=x$ and $\perp(x)=0$ for all $x \in[0,1]$. Note that $i d$ and $\perp$ represent the strongest and the weakest degrees of $f$-inclusion.

Obviously, the greater the mapping $f \in \Omega$ the stronger the restriction imposed by the $f$-inclusion; since given $A \subseteq \subseteq_{f} B$, the value $f(A(u))$ determines a lower bound of the possible values of $B(u)$, for all $u \in \mathscr{U}$. In this way, the degree of inclusion of fuzzy set $A$ into fuzzy set $B$ can be defined by choosing the greatest $f \in \Omega$ such that $A$ is $f$-included in $B$; for more details we refer the reader to [8].

Definition 3 Let $A$ and $B$ be two fuzzy sets, the $f$ degree of inclusion of $A$ in $B$, denoted by $\operatorname{Inc}(A, B)$, is defined as follows

$$
\operatorname{Inc}(A, B)=\max \left\{f \in \Omega \mid A \subseteq_{f} B\right\} .
$$

The following analytical expression of the $f$-degree of inclusion is given in [8]:

$$
\begin{aligned}
\operatorname{Inc}(A, B) & =f_{A, B} \wedge i d \quad \text { where } \\
f_{A, B}(x) & =\bigwedge_{u \in \mathscr{U}}\{B(u) \mid x \leq A(u)\} .
\end{aligned}
$$

The following result summarizes some properties of the $f$-degree of inclusion defined above that resemble some axiomatic approaches of measures of inclusion given in the literature $[7,11]$.

Theorem 1 Let $A, B$ and $C$ be three fuzzy sets, then

1. If $A$ and $B$ are two crisp sets, then either $\operatorname{Inc}(A, B)=$ id or $\operatorname{Inc}(A, B)=\perp$;

2. $\operatorname{Inc}(A, B)=i d$ if and only if $A(u) \leq B(u)$ for all $u \in \mathscr{U}$;

3. If $\mathscr{U}$ is finite, $\operatorname{Inc}(A, B)=\perp$ if and only if there exists $u \in \mathscr{U}$ such that $A(u)=1$ and $B(u)=0$;

4. $\operatorname{Inc}(B, C) \circ \operatorname{Inc}(A, B) \leq \operatorname{Inc}(A, C)$;

5. If $B(u) \leq C(u)$ for all $u \in \mathscr{U}$ then,

(a) $\operatorname{Inc}(A, B) \leq \operatorname{Inc}(A, C)$ and

(b) $\operatorname{Inc}(C, A) \leq \operatorname{Inc}(B, A)$;

6. $\operatorname{Inc}(A, B \cap C)=\operatorname{Inc}(A, B) \wedge \operatorname{Inc}(A, C)$;

7. $\operatorname{Inc}(A \cup B, C)=\operatorname{Inc}(A, C) \wedge \operatorname{Inc}(B, C)$.

\subsection{The notion of $f$-weak contradiction}

As with $f$-inclusion, the underlying idea here is to measure contradiction not with real values, but with certain mappings from $[0,1]$ to $[0,1]$. In this case, those mappings used as degrees of contradiction should satisfy some properties that resemble negation operators.

Definition 4 The set of $f$-degrees of contradiction (denoted by $\bar{\Omega}$ ) is the set of mappings $f:[0,1] \rightarrow[0,1]$ satisfying the following properties for all $x, y \in[0,1]$ :

- $f(0)=1$;

- if $x \leq y$ then $f(y) \leq f(x)$

The notion of $f$-weak-contradiction $[2,3]$ is a generalization of the notion of $\mathrm{N}$-contradiction given by Trillas, Alsina and Jacas [12]

Definition 5 Let $A$ and $B$ be two fuzzy sets defined over a nonempty universe $\mathscr{U}$ and let $f \in \bar{\Omega}$. We say that $A$ is $f$-weak-contradictory w.r.t. $B$ if and only if $A(u) \leq$ $f(B(u))$ holds for all $u \in \mathscr{U}$.

Note that $\bar{\Omega}$ has a structure of complete lattice with the usual pointwise ordering. We denote by $f_{\top}$ and $f_{\perp}$ the greatest and lowest mapping in $\bar{\Omega}$; i.e., the weakest and the strongest degrees of $f$-weak-contradiction; for more details we refer the reader to [3].

Below we summarise some properties of the notion of $f$-weak-contradiction which motivates the consideration of $\bar{\Omega}$ as a proper set of degrees of contradiction.

Theorem 2 Let $A, B$ and $C$ be three fuzzy sets and let $f, g \in \bar{\Omega}$. Then,

1. $A$ is $f_{\perp}$-weak-contradictory w.r.t. $B$ if and only if $B(u)>0$ implies $A(u)=0$ for all $u \in \mathscr{U}$;

2. If $A \leq B$ and $C$ is $f$-weak-contradictory w.r.t. $B$ then, $C$ is $f$-weak-contradictory w.r.t. A,

3. If $A \leq C$ and $C$ is $f$-weak-contradictory w.r.t. $B$ then, $A$ is $f$-weak-contradictory w.r.t. $B$;

4. If $\mathscr{U}$ is a finite universe, $f_{\top}$-weak-contradiction is the only $f$-weak-contradiction of $A$ w.r.t. $B$ if and only if there exists $u \in \mathscr{U}$ such that $A(u)=$ $B(u)=1$;

Notice that, given two fuzzy sets $A$ and $B$, a degree of contradiction between $A$ and $B$ can be defined by choosing the least $f \in \bar{\Omega}$ such that $A$ is $f$-weakcontradictory w.r.t. $B$. 
Definition 6 ( [3]) Let $A$ and $B$ be two fuzzy sets, the $f$-degree of contradiction of $A$ w.r.t. $B$, denoted by Con $(A, B)$, is given by the least mapping $f \in \bar{\Omega}$ verifying that $A$ is $f$-weak-contradictory w.r.t. $B$.

Note that it is necessary to prove that the $f$-degree of contradiction is well-defined, i.e., that the least mapping $f \in \bar{\Omega}$ verifying that $A$ is $f$-weak-contradictory w.r.t. $B$ exists. Such a proof can be found in [3], together with the following analytic expression for $\operatorname{Con}(A, B)(x)$ :

$\operatorname{Con}(A, B)(x)= \begin{cases}1 & \text { if } x=0 \\ \bigvee_{u \in \mathscr{U}}\{A(u) \mid x \leq B(u)\} & \text { otherwise. }\end{cases}$

The similarities between the $f$-degrees of inclusion and the $f$-degree of contradiction are now obvious. The following result is a direct consequence of Theorem 2 and characterizes the two extreme degrees of contradiction, namely $f_{\perp}$ and $f_{\top}$ that represents the greatest and lowest degree of contradiction, respectively.

Corollary 1 Let $A$ and $B$ be two fuzzy sets, then,

1. $\operatorname{Con}(A, B)=f_{\perp}$ if and only if $B(u)>0$ implies $A(u)=0$ for all $u \in \mathscr{U}$.

2. If $\mathscr{U}$ is finite, $\operatorname{Con}(A, B)=f_{\top}$ if and only if there exists $u \in \mathscr{U}$ such that $A(u)=B(u)=1$.

At this point, it is worth noting that the pointwise ordering between the different $f$-degrees of contradiction classifies the $f$-degrees inversely to the strength of the contradiction that they represent. That is, the smaller the function $\operatorname{Con}(A, B)$, the stronger the contradiction of $A$ w.r.t. $B$.

\section{$3 f$-degrees of inclusion/contradiction in the square of opposition}

The classical Aristotelian square of opposition has the following form

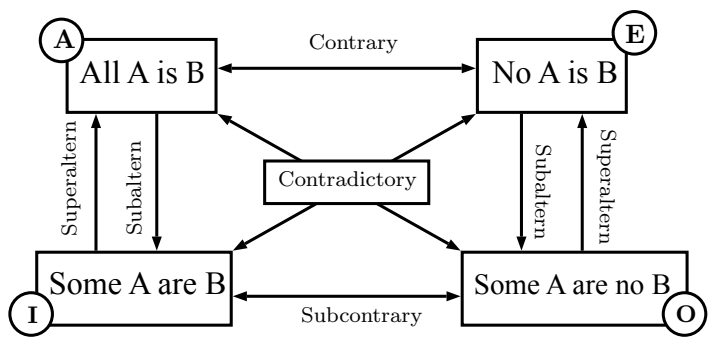

There are four vertexes:
- A, or Universal Affirmative, is represented by the statement "All A is B", and is formulated in firstorder logic as the formula $\forall x(A(x) \rightarrow B(x))$.

- E, or Universal Negation, is represented by the statement "No A is $B$ " and is formulated in firstorder logic as the formula $\forall x(A(x) \rightarrow \neg B(x))$.

- I, or Particular Affirmative, is represented by the statement "Some A is B" and is formulated in first-order logic as the formula $\exists x(A(x) \wedge B(x))$.

- O, or Particular Negation, is represented by "Some A is not B" and is formulated in first-order logic as the formula $\exists x(A(x) \wedge \neg B(x))$.

The four vertexes are connected by different lines (or vectors) representing four different relationships:

- Contradictory: two statements are contradictory if necessarily one must be true and the other false. Note that this relationship is different from the idea represented by the $f$-degree of contradiction.

- Contrary: two statements are contrary if they both cannot be true simultaneously. Note that conversely to contradictory statements, two contrary statements can be false simultaneously.

- Subcontrary: two statements are subcontrary if they both cannot be false simultaneously. Note that conversely to contradictory and contrary statements, two subcontrary statements can be true simultaneously.

- Subaltern: If a statement is true then, its subaltern must be true as well. The ordering of statements here is important. For example, the statement $\mathbf{A}$ is subaltern of $\mathbf{I}$ but $\mathbf{I}$ is not subaltern of $\mathbf{A}$, instead, we say that $\mathbf{I}$ is superaltern of $\mathbf{A}$.

Note now that the vertexes $\mathbf{A}, \mathbf{E}, \mathbf{I}$ and $\mathbf{O}$ can be interpreted in terms of set relations by identifying the (unary) predicates $A$ and $B$ with sets. In this way, we have the following four identifications:

- $\mathbf{A}: A \subseteq B$.

- $\mathbf{E}: A \subseteq B^{c}$

- $\mathbf{I}: A \cap B \neq \varnothing$.

- O: $A \cap B^{c} \neq \varnothing$.

Note that A represents (full) inclusion, $\mathbf{E}$ (full) contradiction, I represents no contradiction, and $\mathbf{O}$ represents no inclusion. Therefore, it makes sense to identify those vertexes with the four extreme $f$-indexes of inclusion and contradiction, namely 


$$
\mathbf{A} \equiv i d \quad \mathbf{O} \equiv \perp \quad \mathbf{E} \equiv f_{\perp} \quad \mathbf{I} \equiv f_{\top} .
$$

Note also that the four first-order formulas that formally represent the four vertexes are also satisfied by such a consideration. By Theorem 1 and Corollary 1 , we have the following for a finite universe $\mathscr{U}$ :

- $\mathbf{A} \equiv i d$ is equivalent to saying that $A(u) \leq B(u)$ for all $u \in \mathscr{U}$, which is equivalent to saying that $\forall u(A(u) \rightarrow B(u))$ has truth degree 1 for any residuated implication $\rightarrow$.

- $\mathbf{E} \equiv f_{\perp}$ is equivalent to saying that $B(u)>0$ implies $A(u)=0$ for all $u \in \mathscr{U}$, which is equivalent to saying that $\forall u(A(u) \rightarrow \neg B(u))$ has truth degree 1 for any residuated implication $\rightarrow$ and any involutive negation operator $\neg$.

- $\mathbf{I} \equiv f_{\top}$ is equivalent to saying that there exists $u \in$ $\mathscr{U}$ such that $A(u)=B(u)=1$, which is equivalent to saying that $\exists u(A(u) \wedge B(u))$ has truth degree 1 for any t-norm that represents $\wedge$.

- $\mathbf{O} \equiv \perp$ is equivalent to saying that there is an element in the universe $u \in \mathscr{U}$ such that $A(u)=1$ and $B(u)=0$, which is equivalent to saying that $\exists u(A(u) \wedge \neg B(u))$ has truth degree 1 for any tnorm that represents $\wedge$ and any involutive negation operator $\neg$.

Following this identification, the rest of $f$-degrees of inclusion and contradiction can be allocated in the diagonal joining the respective vertexes:
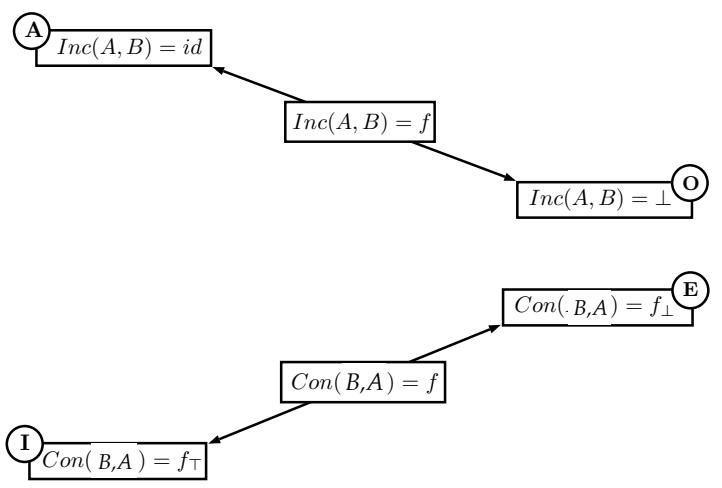

The $f$-degrees of inclusion and contradiction are allocated in order, that is, the stronger the inclusion (resp. contradiction), the closer the respective $f$-degree of inclusion (resp. contradiction) to A (resp. to $\mathbf{E}$ ). However, although in the diagonal joining $\mathbf{A}$ and $\mathbf{O}$ the $f$ degrees are ordered by following the natural order between functions in $[0,1]^{[0,1]}$, in the diagonal joining $\mathbf{E}$ and $\mathbf{I}$ the $f$-degrees are ordered reversely. The reason is because the stronger the contradiction, the lesser the $f$-degree of contradiction. As special cases, note that $\mathbf{E} \equiv f_{\perp}$ and $\mathbf{I} \equiv f_{\top}$ represent the strongest and weakest contradictions.

Under such a representation, the relationship of "contradictory statements" given by the diagonals of the square of opposition holds in the following sense: given two fuzzy sets $A$ and $B$, the $f$-degree of inclusion and the $f$-degree of contradiction are unique. In other words, "contradictory statements" is interpreted as "only one statement can be true and one must be true".

The rest of relationships imposed by the square of opposition (i.e., Contrary, Subcontrary, Subaltern and Superaltern) are more intricate. That is due to the following two circumstances:

- Using $f$-degrees of inclusion and $f$-degrees of contradiction in the diagonals of the square of opposition leads to the fact that we are no longer working with Boolean truth-values, but with degrees. In this way, the inference given in the Aristotelian (crisp) square of opposition by the consideration of "true" and "false" statements has to be reconsidered.

- The other circumstance is related to the (important) fact that the relationships in the crisp square of opposition hold if and only if the set $A$ is not empty, this is the well-known requirement of existential import. In our approach, instead of seeing such a feature as a shortcoming, it is considered as an incentive to determine a degree of emptiness via the $f$-degree of inclusion and contradiction.

Due to the features above, the square of opposition has received some criticisms, but it is still considered as a reference for current research on this topic $[5,6]$. In fact, in those works different opposition structures are studied both from a classical and a fuzzy standpoint.

To begin with, in our approach, let us assume a nonempty environment by considering normal fuzzy sets and let us interpret the conditions imposed by the Contrary, Subaltern/Superaltern relationships into the context of $f$-degrees of inclusion and contradiction. Such relationships define six implications, namely

$$
\begin{array}{lll}
\text { Contraries } & \text { Subalterns } & \text { Superalterns } \\
\text { - } \mathbf{A} \Rightarrow \neg \mathbf{E} & \bullet \mathbf{A} \Rightarrow \mathbf{I} & \bullet \neg \mathbf{I} \Rightarrow \neg \mathbf{A} \\
-\mathbf{E} \Rightarrow \neg \mathbf{A} & \bullet \mathbf{E} \Rightarrow \mathbf{O} & \bullet \neg \mathbf{O} \Rightarrow \neg \mathbf{E}
\end{array}
$$

Note that the statements $\mathbf{A}, \mathbf{E}, \mathbf{I}$ and $\mathbf{O}$ have been identified above with the extreme degrees of inclusion and contradiction. Moreover, they are pairwise contradictory in the square of opposition, i.e., $\mathbf{A}=\neg \mathbf{O}$ and 
$\mathbf{E}=\neg \mathbf{I}$. Therefore, we can go one step further and do the following identification:

- $\mathbf{A}=\neg \mathbf{O}$ as a strong inclusion,

- $\mathbf{E}=\neg \mathbf{I}$ as a strong contradiction,

- $\neg \mathbf{A}=\mathbf{O}$ as a weak inclusion,

- $\neg \mathbf{E}=\mathbf{I}$ as a weak contradiction.

As a result the six previous implications lead to the same interpretation:

the stronger the inclusion, the weaker the contradiction

and the stronger the contradiction, the weaker the inclusion.

In fuzzy set theory, we can interpret that two statements are Contrary if the greater the degree of truth of one, the lesser the degree of truth of the other. In our case, the $f$-indexes are somehow truth degrees of inclusion or contradiction and the following result shows that both indexes are "contrary". Such a kind of monotonicity is given directly below:

Proposition 1 Let $A$ and $B$ be two fuzzy sets such that $A$ is normal, then $\operatorname{Inc}(A, B)<\operatorname{Con}(B, A)$.

Consequently, we obtain that the stronger the inclusion, the greater the $f$-degree of inclusion, the greater the $f$-degree of contradiction and then, the weaker the contradiction. Conversely, we can also say that the stronger the contradiction, the lesser the $f$-degree of inclusion, the lesser the $f$-degree of contradiction and then, the weaker the inclusion. In other words, the $f$ degree of inclusion and the $f$-degree of contradiction are contrary.

The following example shows that the hypothesis $A$ is a normal fuzzy set is necessary to achieve Proposition 1.

Example 1 Let $\mathscr{U}=\{u\}$ and $A(u)=0.5$. Then $\operatorname{Inc}(A, A)=i d$ and

$$
\operatorname{Con}(A, A)(x)= \begin{cases}1 & \text { if } x=0 \\ 0.5 & \text { if } 0<x \leq 0.5 \\ 0 & \text { otherwise. }\end{cases}
$$

Obviously, $\operatorname{Inc}(A, A) \quad \nless \quad \operatorname{Con}(A, A), \quad$ since $\operatorname{Inc}(A, A)(1)=1$ and $\operatorname{Con}(A, A)(1)=0$.

The situation of the previous example is related to the empty set. Note that in fuzzy set theory the notion of empty set can be interpreted in two different ways, the extreme case is by defining the empty fuzzy set $\varnothing$ by $\varnothing(u)=0$ for all $u \in \mathscr{U}$, the most relaxed case is via non-normal fuzzy sets, in which no element completely belongs to the set. In our approach the latter interpretation is the more convenient. Under such a view, a non-normal fuzzy set is considered to be partially empty.

The following proposition shows that if the $f$-degrees of inclusion and contradiction do not satisfy Proposition 1 (i.e., they are not contrary), then one of the fuzzy sets is partially empty.

Proposition 2 Let $A$ and $B$ be two fuzzy sets such that there exists $\alpha \in(0,1]$ satisfying the inequality $\operatorname{Inc}(A, B)(\alpha)>\operatorname{Con}(B, A)(\alpha)$. Then:

$$
\begin{aligned}
& \text { 1. } A(u)<\alpha \text { for all } u \in \mathscr{U} ; \\
& \text { 2. } \operatorname{Inc}(A, B)(x)=x \text { for all } x \geq \alpha \text {; } \\
& \text { 3. } \operatorname{Con}(B, A)(x)=0 \text { for all } x \geq \alpha \text {. }
\end{aligned}
$$

Let us reconsider the case where $A$ is normal and let us analize the properties related to subalterns in terms of the existence of elements in the intersection of fuzzy sets. The first result concerns the $f$-degree of inclusion and the statement $\mathbf{I}$.

Proposition 3 Let $A$ and $B$ be two fuzzy sets such that $A$ is normal, then there exists $u \in \mathscr{U}$ such that $(A \cap$ $B)(u) \geq \operatorname{Inc}(A, B)(1)$.

It is not difficult to check that the statement fails whenever $A \subseteq B$ and $A$ is not normal: in this case, we would have $\operatorname{Inc}(A, B)=i d$ and $A \cap B$ will always be smaller than 1 . Specifically,

Example 2 Consider $A$ as in Example 1, and $B=A$. Then, $(A \cap B)(u)=0.5$ but $\operatorname{Inc}(A, B)(1)=1$.

Note that the previous result determines a subaltern relationship concerning the $f$-degree of inclusion. Specifically, we can determine a least bound for the least truth degree of the intersection of two fuzzy sets (as the statement $\mathbf{I}$ ) by the $f$-degree of inclusion. Moreover, the greater the $f$-degree of inclusion, the less empty the intersection.

In the case of the $f$-degree of contradiction we have two different possibilities to understand the subaltern relationship.

Proposition 4 Let $A$ and $B$ be two fuzzy sets such that $A$ is normal, then there exists $u \in \mathscr{U}$ such that $(A \cap$ $B)(u) \leq \operatorname{Con}(B, A)(1)$.

The following result is written in a form similar to the existential first-order formula that defines formally $\mathbf{O}$. 
Proposition 5 Let $A$ and $B$ be two fuzzy sets such that $A$ is normal and let $n$ be an involutive negation. Then there exists $u \in \mathscr{U}$ such that $\left(A \cap B^{c}\right)(u) \geq$ $n(\operatorname{Con}(B, A)(1))$.

Once again, Example 1 provides counterexamples for the case of $A$ not being a normal fuzzy set.

The interpretation of the previous results as the subaltern relationship is equivalent to the one made for Proposition 3. Moreover, as a consequence of the previous results, we have the classical subaltern and superaltern relationships between the extreme cases of inclusion and contradiction.

Corollary 2 Let $A$ and $B$ be two fuzzy sets such that $A$ is normal, then:

- if $\operatorname{Inc}(A, B)=$ id then, $\operatorname{Con}(A, B)=f_{\top}$;

- if $\operatorname{Con}(A, B)=f_{\perp}$ then, $\operatorname{Inc}(A, B)=\perp$.

To end this section, let us see the square of opposition in terms of the $f$-degrees of inclusion and contradiction. Let us asume that $A$ and $B$ are two fuzzy sets, that the $f$-degree of inclusion is unknown and that the $f$ degree of contradiction is known, say $\operatorname{Con}(B, A)=f$. Then, we can allocate $\operatorname{Con}(B, A)=f$ in the respective diagonal and since such a diagonal represents a contradictory relationship, we can conclude that the rest of $f$-degree of contradiction does not hold. Then, we have the following diagram:

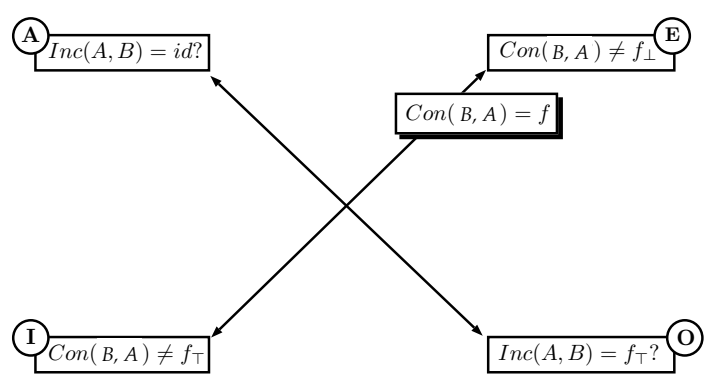

Note that the preliminary lack of knowledge about $\operatorname{Inc}(A, B)$ is represented in the diagram above by the symbol "? ". However, such a lack of knowledge is partial, since we can use that $\operatorname{Inc}(A, B)$ and $\operatorname{Con}(B, A)$ are contrary and apply Proposition 1 . This can be represented in the diagram as follows:

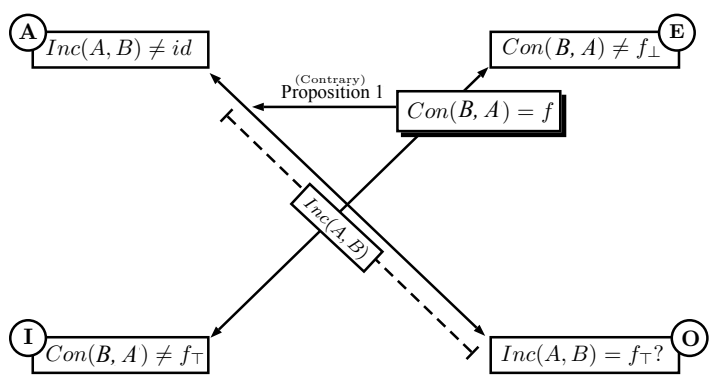

which bound the range of possible values for $\operatorname{Inc}(A, B)$ and moreover, discard the strongest degree of inclusion $\operatorname{Inc}(A, B)=i d$. Finally, we can use also the subaltern property and approximate a truth-value for the formula $\exists x(A(x) \cap \neg B(x))$ by Proposition 5. We can represent such an inference in the diagram by a statement denoted by $\mathbf{O}^{*}$

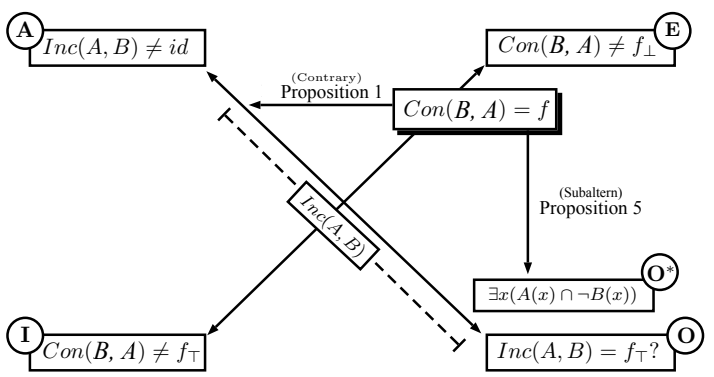

A similar reasoning starting from the $f$-degree of inclusion can be performed.

\section{Conclusions and future works}

We have used the $f$-degrees of inclusion and contradiction to approach the square of opposition from a generalized standpoint. The Boolean case allows, given knowledge about one of the vertices, to infer information about some of the other vertices; in our case the idea is essentially the same.

Our approach to inclusion and contradiction is gradual, and each of these notions translate to one diagonal. Once we know, for instance, the degree of contradiction, it would be located somewhere in the corresponding diagonal, then by Proposition 1 we obtain an upper bound for the degree of inclusion and, finally, Proposition 5 somehow allows reasoning by subaltern, and ensures the existence of an element satisfying certain properties.

Further work in this line will focus on trying to relate our work with other approaches. On the one hand, one 
can find gradual versions of the square (and other structures) of opposition in the literature: in [6] the square is in fact a 4-tuple of truth-degrees which should fulfill certain relations among them in order to properly generalize the different notions of opposition, in [10] graded structures of opposition are used in fuzzy natural logic. On the other hand, in this paper, we identify the segment AO with the index of inclusion, and the segment EI as the index of contradiction, and the obtained results allow extracting information in terms of opposition.

Another possible direction for future research is based on the possible consequences of the obtained results in terms of formal concept analysis, following the ideas in $[1,4]$.

\section{Acknowledgements}

Partially supported by the Ministry of Science, Innovation, and Universities (MCIU), the State Agency of Research (AEI) and the European Social Fund (FEDER) through the research project PGC2018-095869B-I00 (MCIU/AEI/FEDER, UE), and by Junta de Andalucía, Universidad de Málaga and the European Social Fund (FEDER) through the research project UMA2018FEDERJA-001.

We also want to thank the reviewers for helping to improve the original submitted version.

\section{References}

[1] S. Boffa, P. Murinová, and V. Novák Graded polygons of opposition in Fuzzy Formal Concept Analysis. International Journal of Approximate Reasoning, 132:128-153, 2021.

[2] H. Bustince, N. Madrid, and M. Ojeda-Aciego. A measure of contradiction based on the notion of n-weak-contradiction. In IEEE Intl Conf on Fuzzy Systems (FUZZ-IEEE'13), pages 1-6, 2013.

[3] H. Bustince, N. Madrid, and M. Ojeda-Aciego. The notion of weak-contradiction: definition and measures. IEEE Transactions on Fuzzy Systems, 23:1057-1069, 2015.
[4] Dubois, D., Prade, H.. From Blanche's hexagonal organization of concepts to formal concepts analysis and possibility theory. Logica Universalis 6:149-169, 2012.

[5] D. Dubois, H. Prade, and A. Rico. Graded cubes of opposition and possibility theory with fuzzy events. Int. J. of Approximate Reasoning, 84:168-185, 2017.

[6] D. Dubois, H. Prade, and A. Rico. Structures of opposition and comparisons: Boolean and gradual cases. Logica Universalis, 14:115-149, 2020.

[7] L. M. Kitainik. Fuzzy inclusions and fuzzy dichotomous decision procedures. In J. Kacprzyk and S. A. Orlovski, editors, Optimization Models Using Fuzzy Sets and Possibility Theory, pages 154-170. Springer Netherlands, Dordrecht, 1987.

[8] N. Madrid and M. Ojeda-Aciego. Functional degrees of inclusion and similarity between L-fuzzy sets. Fuzzy Sets and Systems 390:1-22, 2020.

[9] N. Madrid, M. Ojeda-Aciego, and I. Perfilieva. finclusion indexes between fuzzy sets. In Proc. of IFSA-EUSFLAT, 2015.

[10] P. Murinová. Graded Structures of Opposition in Fuzzy Natural Logic. Logica Universalis 265:495-522, 2022.

[11] D. Sinha and E. Dougherty. Fuzzification of set inclusion: Theory and applications. Fuzzy Sets and Systems, 55:15-42, 1993.

[12] E. Trillas, C. Alsina, and J. Jacas. On contradiction in fuzzy logic. Soft Computing, 3(4):197199, 1999.

[13] B. Xie, L.-W. Han, and J.-S. Mi. Measuring inclusion, similarity and compatibility between Atanassov's intuitionistic fuzzy sets. International Journal of Uncertainty, Fuzziness and Knowledge-Based Systems, 20(02):261-280, 2012.

[14] V. Young. Fuzzy subsethood. Fuzzy Sets and Systems, 77:371-384, 1996. 